\title{
Association between Types of Usual Source of Care and User Perception of Overall Health Care Service Quality in Korea
}

\author{
Nak-Jin Sung ${ }^{1}$, Jae-Ho Lee ${ }^{2 * *}$ \\ 'Department of Family Medicine, Dongguk University Ilsan Hospital, Dongguk University College of Medicine, Goyang, Korea \\ ${ }^{2}$ Department of Family Medicine, College of Medicine, The Catholic University of Korea, Seoul, Korea
}

\begin{abstract}
Background: Patients' perceptions of care tend to correlate with the quality of care provided. Different health care systems and service environments may show different associations between types of usual source of care (USC) and overall service quality assessment. We attempted to analyze this association as a benefit of having a USC.

Methods: This study used the 2012 Korea Health Panel data version 1.1 as representative national household survey data. The total number of subjects aged 18 years or more was 12,708 . The number of subjects in the final analysis was 10,665. Multiple logistic regression analysis was used to assess the association between types of USC and overall health care service quality. The main outcome variable was users' ratings of the quality of health care service.

Results: People having a usual doctor $(n=1,796)$ were more likely to positively assess the quality of health care they received than those not having a USC ( $\mathrm{n}=7,920$; odds ratio [OR], 1.39; 95\% confidence interval [CI], 1.20-1.60) or with those having only a place as a USC without a usual doctor (n=949; OR, 1.29; 95\% CI, 1.05-1.58) after adjustment for demographic characteristics and health-related variables.

Conclusion: People having a usual doctor rated overall health care service quality as high, which might be due to benefits of primary care attributes related to usual doctors. Further studies are needed to elucidate the causal relationship. This finding implies that health policies encouraging people to have a usual doctor are needed in Korea.
\end{abstract}

Keywords: Health Care Quality Assessment; Surveys and Questionnaires; Primary Health Care; Korea; Usual Source of Care

Received: July 12, 2017, Revised: August 28, 2017, Accepted: October 12, 2017

*Corresponding Author: Jae-Ho Lee https://orcid.org/0000-0001-5184-7437

Tel: +82-2-2258-6288, Fax: +82-2-2258-2907, E-mail: jaeholee@catholic.ac.kr 


\section{INTRODUCTION}

Usual source of care (USC) refers to the provider or place a patient consults when sick or in need of medical advice. ${ }^{1)}$ USC is closely associated with the four major attributes of primary care: longitudinality, first contact, comprehensiveness, and coordination of care. ${ }^{2)}$ Having a USC has been associated with increased access to health care or health education, more use of preventive services, decreased number of visits to the emergency room, improved health status, and increased satisfaction with health care. Indeed, identification of a particular practitioner of care (a usual doctor) has been associated with better services, such as better recognition of problems or needs, more accurate or earlier diagnoses, a lower rate of emergency care use, fewer hospitalizations, lower costs, better monitoring, fewer drug prescriptions, fewer unmet needs, and increased satisfaction compared with mere identification of a particular place of care (a usual place only). Having a particular place is as good as having a particular doctor only in terms of appointment keeping and for preventive care needed by children at defined times, compared with having no place at all. ${ }^{3-6)}$

Perception of health care service quality is significantly and positively related to patients' satisfaction and their behavioral intentions. ${ }^{7)}$ Among the many assessment methods of health care service quality, users' ratings of quality of care as a type of subjective assessment are unique in that they may include users' overall satisfaction with care, their communication with their care providers, and their ability to obtain needed care quickly. ${ }^{8)}$ Users' ratings may influence future decisions on accessing care and thus form a part of the feedback loop. ${ }^{9)}$

Studies have dealt with the relationship between USC and ratings of overall quality of care. ${ }^{10-12)}$ Individuals with a usual place indicated higher quality of medical care experiences compared with those without a USC, as reported in studies conducted in Taiwan and China. Similarly, in studies conducted in China and the United States, individuals with a usual doctor reported higher quality of medical care experiences compared with those without a USC. In the aforementioned studies conducted in Taiwan and China, patients at medical institutions assessed the quality of their primary care physicians. ${ }^{10,11)}$ Meanwhile, community residents rated the quality of overall health care received in the last 12 months in the study conducted in the United States. $^{12)}$

Korea has a health care system with weak primary care. Strengthening the primary care system is hampered by private sector dominance ( $>90 \%$ ) in health care institutions, political negligence of primary care, and long-standing fee-for-service (FFS) payment schemes, in spite of services of the national health insurance (NHI) system since 1989. Koreans are not required to have a USC. In 2002, only approximately onethird of Koreans have a USC (a usual place or a usual doctor). ${ }^{13,14)}$ Koreans can visit any specialist or any medical institution freely in almost all cases.

Korea, Taiwan, China, and the United States have a weak primary care in common, compared with developed European countries such as the United Kingdom and the Netherlands. However, Korea has some notable differences with the other three nations. Korea and Taiwan have universal health insurance coverage, whereas China and the United States do not. The NHI of Korea has mainly adopted an FFS payment system, whereas Taiwan is expanding the use of diagnosisrelated group payments for hospitals, experimenting with pay-for-performance, case payment, and capitation schemes. ${ }^{15)}$ In this context, Korea might show a different association between USC and user ratings of quality of care with the other nations, as user ratings of quality of care could be influenced by people's preferences of care, the health care system, and the cultures of nations. ${ }^{16)}$ Korea, with universal health insurance coverage and free choice of medical providers, is a better place for a study on the association between USC and quality of care compared with the United States, where it may be difficult to ensure whether the observed effects on medical care experiences are due to USC or insurance coverage. ${ }^{10,17)}$ In addition, much evidence about the benefits of primary care is presently required to suggest health policy for strengthening primary care in Korea. Therefore, the present study attempted to analyze the association between types of USC and people's ratings of overall quality of care in the Korean health care context as a benefit of having a USC using data from a nationally representative sample.

\section{METHODS}

\section{Data and Study Subjects}

Data were derived from the Korea Health Panel (KHP) version 1.1 (2008-2012). Prepared under the auspices of the National Health In-

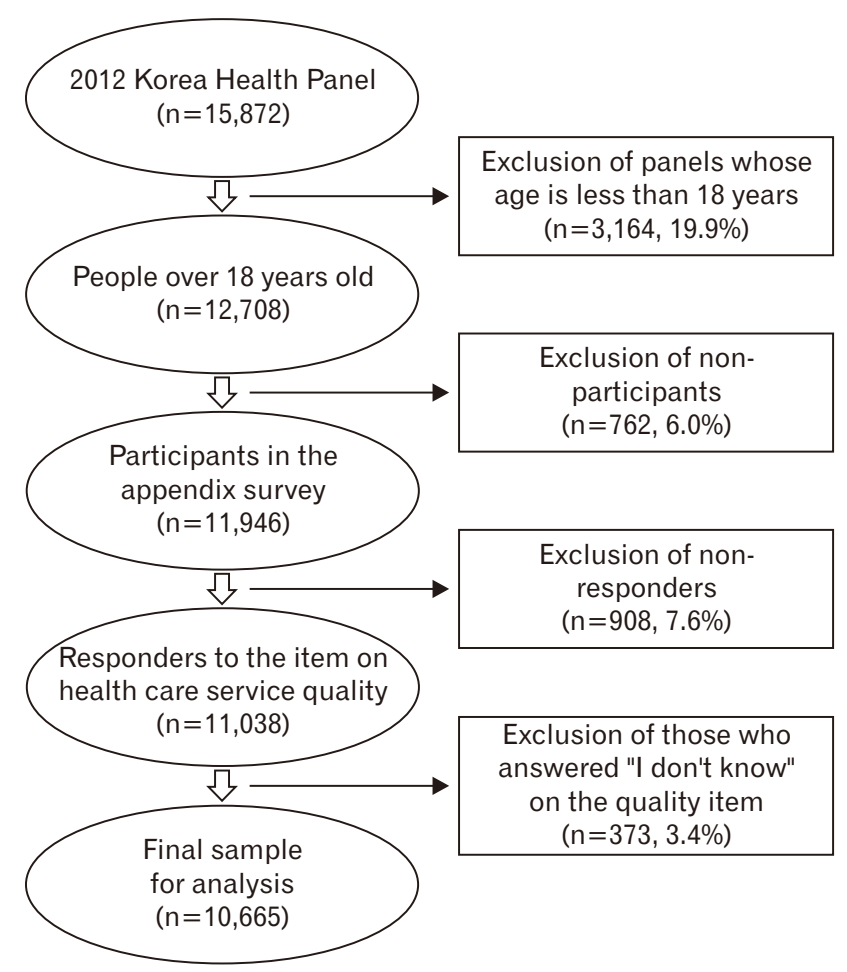

Figure 1. Sample selection process. 
surance Service and the Korea Institute for Health and Social Affairs, the KHP survey provides nationally representative and longitudinal data, covering health status, medical conditions, health care utilization, and health care expenditures. The KHP survey has been administered annually since 2008 using an interview research method in which an interviewer asks questions and records the answers of interviewees. A stratified cluster systematic method was used for selecting a sample of households for the present research. The appendix questions of the 2012 KHP survey, which only persons aged 18 years or older needed to answer, included items on the presence of a USC and a usual doctor, and overall quality of health care service. ${ }^{18)}$ The total number of subjects aged 18 years or older in the 2012 KHP data was 12,708 . The number of subjects in the final analysis was 10,665 (Figure 1).

This study was approved by the Institutional Review Board of the Catholic University of Korea (IRB approval no., KC15QISI0089) with a waiver for informed consent because the data were obtained from a public database (https://www.khp.re.kr:444/).

\section{Variables}

1) Outcome variable: perception of overall health care quality

The question used to assess the quality of medical care was as follows: "Overall, how do you rate the services quality in the medical care that you (or your family) have received in the past 12 months?" The answer choices were "excellent," "good," "fair," "poor," and "I don't know."

The answers were collapsed into two categories of high quality (excellent and good) and low quality (fair and poor), because a relatively small number of people $(n=326,3.1 \%)$ chose the most extreme answers. The "I don't know" answers were excluded from analysis (Figure 1).

\section{2) Predictor variables}

The main variable of interest was types of USC. USCs were classified into the categories of "not having," "having a place only," and "having a usual doctor with or without a place," using data from the following two questions: "Is there a medical institution to which you usually go,

Table 1. Characteristics of study subjects by type of USC (2012 Korea Health Panel)

\begin{tabular}{|c|c|c|c|c|c|}
\hline \multirow{2}{*}{ Characteristic } & \multicolumn{4}{|c|}{ USC } & \multirow{2}{*}{ P-value } \\
\hline & Total & No USC & Usual place only* & Usual doctor & \\
\hline No. of subjects & 10,665 & $7,920(74.3)$ & $949(8.9)$ & $1,796(16.8)$ & \\
\hline Sex & & & & & $<0.001$ \\
\hline Male & 4,795 & $3,690(77.0)$ & $395(8.2)$ & $710(14.8)$ & \\
\hline Female & 5,870 & $4,230(72.1)$ & $554(9.4)$ & $1,086(18.5)$ & \\
\hline Age (y) & & & & & $<0.001$ \\
\hline $18-39$ & 2,834 & $2,532(72.1)$ & $130(4.6)$ & $172(6.1)$ & \\
\hline $40-64$ & 5,174 & $3,926(75.9)$ & $420(8.1)$ & $828(16.0)$ & \\
\hline$\geq 65$ & 2,657 & $1,462(55.0)$ & $399(15.0)$ & $796(30.0)$ & \\
\hline Marital status & & & & & $<0.001$ \\
\hline Married & 7,730 & $5,609(72.6)$ & $721(9.3)$ & $1,400(18.1)$ & \\
\hline Separated, divorced, or widowed & 1,302 & $816(62.7)$ & $162(12.4)$ & $324(24.9)$ & \\
\hline Not married & 1,633 & $1,495(91.6)$ & $66(4.0)$ & $72(4.4)$ & \\
\hline Education (y) & & & & & $<0.001$ \\
\hline $0-6$ & 2,319 & $1,371(59.1)$ & $324(14.0)$ & $624(26.9)$ & \\
\hline $7-12$ & 4,654 & $3,443(74.0)$ & $399(8.6)$ & $812(17.5)$ & \\
\hline$\geq 13$ & 3,692 & $3,106(84.1)$ & $226(6.1)$ & $360(9.8)$ & \\
\hline Household income ${ }^{\dagger}$ (quintile) & & & & & $<0.001$ \\
\hline First (lowest) & 1,609 & $916(56.9)$ & $223(13.9)$ & $470(29.2)$ & \\
\hline Second & 2,030 & $1,511(74.4)$ & $170(8.4)$ & $349(17.2)$ & \\
\hline Third & 2,260 & $1,704(75.4)$ & $185(8.2)$ & $371(16.4)$ & \\
\hline Fourth & 2,331 & $1,837(78.8)$ & $189(8.1)$ & $305(13.1)$ & \\
\hline Fifth (highest) & 2,425 & $1,945(80.2)$ & $182(7.5)$ & $298(12.3)$ & \\
\hline Self-rated health & & & & & $<0.001$ \\
\hline Poor & 1,698 & $1,008(59.4)$ & $248(14.6)$ & $442(26.0)$ & \\
\hline Moderate & 4,271 & $3,159(74.0)$ & $385(9.0)$ & $727(17.0)$ & \\
\hline Good & 4,696 & $3,753(79.9)$ & $316(6.7)$ & $627(13.4)$ & \\
\hline Health coverage & & & & & $<0.001$ \\
\hline National health insurance & 10,167 & $7,658(75.3)$ & $868(8.5)$ & $1,641(16.1)$ & \\
\hline Medical aid & 498 & $262(52.6)$ & $81(16.3)$ & $155(31.1)$ & \\
\hline Outpatient department visits (/y) & $18.6 \pm 26.5$ & $14.6 \pm 22.0$ & $28.3 \pm 32.2$ & $31.3 \pm 34.9$ & $<0.001$ \\
\hline No. of chronic diseases & $1.9 \pm 2.3$ & $1.5 \pm 2.0$ & $3.1 \pm 2.4$ & $3.2 \pm 2.5$ & $<0.001$ \\
\hline
\end{tabular}

Values are presented as number (\%) or mean \pm standard deviation. P-values were based on chi-square tests or analysis of variance with Tukey's method for individual difference; cross-sectional weights for the sampled population were applied.

USC, usual source of care.

${ }^{*}$ A place as a USC without a usual doctor. 'Total household income divided by the square root of the number of household members. 
when you are sick or you want to take tests or consult a doctor?" and "Is there a medical doctor whom you usually see, when you are sick or you want to take tests?"

We selected the other control variables out of the 2012 KHP survey questionnaires after reviewing related articles and considering Korean

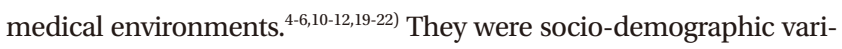
ables (i.e., sex, age, education, total yearly household income, and types of health coverage) and health status variables (i.e., self-rated health status, number of chronic diseases, and number of outpatient visits a year). Total household income was divided by the square root of the numbers of people in the household and then categorized into quintiles. ${ }^{23,24)}$ The question on self-rated health status was originally answered on a 5-point Likert scale and was then collapsed into three categories: poor, moderate, and good.

\section{Statistical Analysis}

Chi-square tests and analysis of variance with Tukey's method for individual difference were used to assess the association between subject characteristics and USC types (i.e., no USC, usual place only, and usual doctor). Chi-square tests and Student t-tests were used to assess the association between subject characteristics and overall health care service quality. Multiple logistic regression analysis was used to assess the association between USC types and overall health care service quality after adjusting for socio-demographic and health status variables. First, those without a USC were set as a reference group (model I). Second, those having a place only were set as a reference group to assess for significant differences between those having a place only and those having a usual doctor (model IA). The Hosmer-Lemeshow test was applied to determine the goodness of fit of the logistic regres-

Table 2. Characteristics of study subjects and assessment of health care service quality (2012 Korea Health Panel)

\begin{tabular}{|c|c|c|c|c|}
\hline \multirow{2}{*}{ Characteristic } & \multicolumn{3}{|c|}{ Health care service quality } & \multirow{2}{*}{ P-value } \\
\hline & Total & Low & High & \\
\hline No. of subjects & 10,665 & $2,637(24.7)$ & $8,028(75.3)$ & \\
\hline USC & & & & $<0.001$ \\
\hline No USC & 7,920 & $2,124(26.8)$ & $5,796(73.2)$ & \\
\hline Usual place only* & 949 & $193(20.3)$ & $756(79.7)$ & \\
\hline Usual doctor & 1,796 & $320(17.8)$ & 1,476 (82.2) & \\
\hline Sex & & & & $<0.001$ \\
\hline Male & 4,795 & 1,266 (26.4) & $3,529(73.6)$ & \\
\hline Female & 5,870 & $1,371(23.4)$ & $4,499(76.6)$ & \\
\hline Age (y) & & & & $<0.001$ \\
\hline $18-39$ & 2,834 & $871(30.7)$ & $1,963(69.3)$ & \\
\hline $40-64$ & 5,174 & $1,383(26.7)$ & $3,791(73.3)$ & \\
\hline$\geq 65$ & 2,657 & $383(14.4)$ & $2,274(85.6)$ & \\
\hline Marital status & & & & $<0.001$ \\
\hline Married & 7,730 & $1,959(25.3)$ & $5,771(74.7)$ & \\
\hline Separated, divorced, or widowed & 1,302 & $194(14.9)$ & $1,108(85.1)$ & \\
\hline Not married & 1,633 & $484(29.6)$ & $1,149(70.4)$ & \\
\hline Education (y) & & & & $<0.001$ \\
\hline $0-6$ & 2,319 & $332(14.3)$ & $1,987(85.7)$ & \\
\hline $7-12$ & 4,654 & $1,212(26.0)$ & $3,442(74.0)$ & \\
\hline$\geq 13$ & 3,692 & 1,093 (29.6) & $2,599(70.4)$ & \\
\hline Household income ${ }^{\dagger}$ (quintile) & & & & $<0.001$ \\
\hline First (lowest) & 1,609 & $269(16.7)$ & $1,340(83.3)$ & \\
\hline Second & 2,030 & $533(26.3)$ & $1,497(73.7)$ & \\
\hline Third & 2,260 & $598(26.5)$ & $1,662(73.5)$ & \\
\hline Fourth & 2,331 & $611(26.2)$ & $1,720(73.8)$ & \\
\hline Fifth (highest) & 2,425 & $621(25.6)$ & $1,804(74.4)$ & \\
\hline Self-rated health & & & & $<0.001$ \\
\hline Poor & 1,698 & $393(23.1)$ & $1,305(76.9)$ & \\
\hline Moderate & 4,271 & $1,148(26.9)$ & $3,123(73.1)$ & \\
\hline Good & 4,696 & $1,096(23.3)$ & $3,600(76.7)$ & \\
\hline Health coverage & & & & $<0.001$ \\
\hline National health insurance & 10,167 & $2,565(25.2)$ & $7,602(74.8)$ & \\
\hline Medical aid & 498 & $72(14.5)$ & $426(85.5)$ & \\
\hline Outpatient department visits (/y) & $18.6 \pm 26.5$ & $15.3 \pm 22.5$ & $19.7 \pm 27.6$ & $<0.001$ \\
\hline No. of chronic diseases & $1.9 \pm 2.3$ & $1.58 \pm 2.1$ & $2.0 \pm 2.3$ & $<0.001$ \\
\hline
\end{tabular}

Values are presented as number (\%) or mean \pm standard deviation. P-values were based on chi-square tests or Student t-tests; cross-sectional weights for the sampled population were applied.

USC, usual source of care.

${ }^{*}$ A place as a USC without a usual doctor. ${ }^{\dagger}$ Total household income divided by the square root of the number of household members. 
sion model. The discriminative ability of the model was assessed using the concordance (C) statistic, the area under the receiver operating characteristic curve. $\mathrm{C}$ statistics generally range from 0.5 (random concordance) to 1 (perfect concordance). ${ }^{25)}$ Stata SE ver. 14.0 (Stata Corp., College Station, TX, USA) was used for statistical analysis, with $\mathrm{P} \leq 0.05$ regarded as a significant difference. Sampling weights were applied in all analyses when calculating the P-values.

\section{RESULTS}

Of the study subjects, women were more likely to have usual doctors than men (18.5\% versus $14.8 \%$ ). The percentage of people having a usual doctor was $30 \%$ among those aged 65 years or more, $6.1 \%$ among those aged $18-39$ years, $18.1 \%$ for those who lived with their spouse, and $24.9 \%$ for those who were separated or divorced. Those with 6 years or less of education had usual doctors more often compared with those with 13 years or more (26.9\% versus $9.8 \%$ ). Those who assessed their health status as poor were more likely to have usual doctors compared with those who believed they were in good health (26.0\% versus $13.4 \%$ ) (Table 1 ).

Respondents having a usual doctor rated health care service quality higher compared with those without a USC (82.2\% versus $73.2 \%)$. Women were likely to assess health care service quality as high compared with men (76.6\% versus $73.6 \%$ ). The percentage of those who perceived health care service quality as high was higher in those aged 65 years and over compared with those aged $18-39$ years ( $85.6 \%$ versus $69.3 \%)$, in those divorced or separated than in those living with spouse (85.1\% versus $74.7 \%$ ), among those educated for 6 years or less than among those educated for 13 years or more (85.7\% versus $70.4 \%$ ), and in those in the lowest quintile than those in the highest quintile of total household income (83.3\% versus $74.4 \%$ ) (Table 2 ).

In the logistic regression analysis adjusted for socio-demographic factors (sex, age, marital status, education, total household income, and health coverage type) and health-related variables (self-rated health status, number of chronic diseases, and number of outpatient visits a year), the odds ratios (ORs) of giving a good assessment to health care were 1.39 (95\% confidence interval [CI], 1.20-1.60) among those with a usual doctor and 1.08 (95\% CI, 0.91-1.28) among those having a usual place only, compared with those without a USC, in model I; they were 1.29 (95\% CI, 1.05-1.58) in those with a usual doctor, compared with those having a usual place of care only, in model IA (Table 3). In this model, Hosmer-Lemeshow tests of goodness of fit resulted in $\mathrm{P}=0.86$. The discriminative ability of this model assessed using the concordance statistic was $\mathrm{C}=0.61$.

In models I and IA, the ORs for assessing overall health care service as high were 2.22 (95\% CI, 1.79-2.75) in the group of people aged 65 years or older (versus the group aged 18-39 years); 1.80 (95\% CI, 1.542.10 ) in the good (versus poor) self-rated health groups; 1.55 (95\% CI, 1.17-2.07) among medical aid beneficiaries (versus people with NHI coverage); 0.56 (95\% CI, 0.47-0.68) in those with 13 years or more of education, and 0.62 (95\% CI, 0.52-0.74) in those with 7-12 years of ed- ucation, compared with those with 6 years or less of education; and 0.82 (95\% CI, 0.68-0.99) for those in the second quintile (versus the first quintile) in total household income. The number of chronic diseases did not have a significant effect on individual perception of overall health care service quality (Table 3 ).

\section{DISCUSSION}

The quality of medical services has been defined and measured in various ways. Users' evaluation, one of many quality measures, is re-

Table 3. ORs of high assessment of health care service quality (2012 Korea Health Panel)

\begin{tabular}{|c|c|c|}
\hline Characteristic & Model I* & Model I $\mathrm{A}^{\dagger}$ \\
\hline \multicolumn{3}{|l|}{ USC } \\
\hline No USC & 1.00 & $0.93(0.78-1.10)$ \\
\hline Usual place only ${ }^{\ddagger}$ & $1.08(0.91-1.28)$ & 1.00 \\
\hline Usual doctor & $1.39(1.20-1.60)$ & $1.29(1.05-1.58)$ \\
\hline \multicolumn{3}{|l|}{ Sex } \\
\hline Male & 1.00 & 1.00 \\
\hline Female & $1.10(1.01-1.21)$ & $1.10(1.01-1.21)$ \\
\hline \multicolumn{3}{|l|}{ Age (y) } \\
\hline $18-39$ & 1.00 & 1.00 \\
\hline $40-64$ & $1.19(1.05-1.35)$ & $1.19(1.05-1.35)$ \\
\hline$\geq 65$ & $2.22(1.79-2.75)$ & $2.22(1.79-2.75)$ \\
\hline \multicolumn{3}{|l|}{ Marital status } \\
\hline Married & 1.00 & 1.00 \\
\hline Separated, divorced, or widowed & $1.23(1.02-1.48)$ & $1.23(1.02-1.48)$ \\
\hline Not married & $1.10(0.96-1.26)$ & $1.10(0.96-1.26)$ \\
\hline \multicolumn{3}{|l|}{ Education (y) } \\
\hline $0-6$ & 1.00 & 1.00 \\
\hline $7-12$ & $0.62(0.52-0.74)$ & $0.62(0.52-0.74)$ \\
\hline$\geq 13$ & $0.56(0.47-0.68)$ & $0.56(0.47-0.68)$ \\
\hline \multicolumn{3}{|l|}{ Household income (quintile) $^{\text {. }}$} \\
\hline First (lowest) & 1.00 & 1.00 \\
\hline Second & $0.82(0.68-0.99)$ & $0.82(0.68-0.99)$ \\
\hline Third & $0.95(0.78-1.15)$ & $0.95(0.78-1.15)$ \\
\hline Fourth & $1.07(0.88-1.30)$ & $1.07(0.88-1.30)$ \\
\hline Fifth (highest) & $1.18(0.97-1.43)$ & $1.18(0.97-1.43)$ \\
\hline \multicolumn{3}{|l|}{ Self-rated health } \\
\hline Poor & 1.00 & 1.00 \\
\hline Moderate & $1.26(1.08-1.46)$ & $1.26(1.08-1.46)$ \\
\hline Good & $1.80(1.54-2.10)$ & $1.80(1.54-2.10)$ \\
\hline \multicolumn{3}{|l|}{ Health coverage } \\
\hline National health insurance & 1.00 & 1.00 \\
\hline Medical aid & $1.55(1.17-2.07)$ & $1.55(1.17-2.07)$ \\
\hline Outpatient department visits (/y) & $1.00(1.00-1.00)^{\|}$ & $1.00(1.00-1.00)^{\prime \prime}$ \\
\hline No. of chronic diseases & $0.98(0.95-1.01)$ & $0.98(0.95-1.01)$ \\
\hline
\end{tabular}

Values are presented as odds ratio (95\% confidence interval). Multiple logistic regression analysis. P-value for Hosmer-Lemeshow tests of goodness of fit was 0.86 . The discriminative ability of this model assessed using the concordance statistic was 0.61 . Cross-sectional weights for the sampled population were applied in all analyses when calculating P-values.

USC, usual source of care.

*In model I, P for trend of the main variable of interest, the usual source of care, was less than 0.001 . 'Model IA was the same as model I except that the reference group was changed from no-USC to usual place only. ${ }^{\ddagger} A$ place as a USC without a usual doctor. ${ }^{\S}$ Total household income divided by the square root of the number of household members. "(0.998-1.003). 
lated to overall satisfaction and behavioral intention to use the same health care service again. ${ }^{7)}$ In the present study, people having a usual doctor had a better perception of overall health care service quality compared with those without a USC or those having only a usual place. However, people having only a usual place as a USC without a usual doctor did not show a significant difference in their perception of medical service quality in Korea, compared with those without a USC.

To our knowledge, this study is the first in Korea to analyze the association between having a usual doctor (versus no USC or having only a place) as a USC and overall ratings of health care using a nationally representative data. Our findings serve as evidence that having a usual doctor would make individuals perceive the quality of health care higher compared with having only a usual place or no USC.

A US study, in which the data were collected using a self-administered mailed questionnaire from October 2011 to February 2012, reported that individuals with a usual provider tend to have higher ratings of overall quality of care. ${ }^{12)}$ The authors showed the importance of patient-centered communication in shaping patients' perceptions of the quality of their care, accounting for a significant portion of the observed relation between having a usual provider of care and ratings of quality. The effect of patient-centered communication on health care quality could be true of the medical system and environment in Korea as well, because Korean health care services are fragmented by a short consultation time and delivered mainly in a doctor-oriented manner. The results and method of the above US study were similar to ours in that both studies used a nationally representative survey, one-item global ratings of the quality of health care in the last 12 months, and a Likert scale for assessing service quality. However, the US study included other health professionals apart from a doctor as a usual provider of care, and could not compare the effect between a usual place and a usual provider because of the absence of a "no usual place" item in the questionnaire, in contrast with our study.

Studies conducted in other East Asian countries have shown similar findings with ours. In a Taiwanese study, having a USC (a usual place or a usual doctor) was associated with higher quality of primary care. ${ }^{10)}$ Having either a usual place or a usual doctor was also associated with higher quality of primary care in a Chinese study. ${ }^{11)}$ The above two studies were a little different from ours in that patients assessed the quality of primary care rather than overall quality of health care. In these studies, the total quality scores were obtained by adding up the scores of 34 questionnaire items of the primary care assessment tool. Another difference between our research and the abovementioned Chinese study is that in the latter, patients with a usual place showed higher total scores than those without a usual place. Nonetheless, the ratings of health care quality between people with and without a usual provider were significantly different irrespective of the proportions of people who had a usual doctor $(80 \%$ in the United States, $54 \%$ in China, and $13.9 \%$ in Korea). ${ }^{11,14,26)}$

Mainous and Gill $^{19)}$ analyzed Delaware Medicaid patient data and reported that patients in the high clinician continuity group had lower odds of hospitalization than those in the high site/low clinician continuity group; the latter group did not differ in hospitalization rate from the low site/low clinician continuity group. Blewett et al. ${ }^{4}$ showed that having both a usual place and a usual provider is consistently associated with increased odds for receiving preventive care/screening services compared with having a usual place only or neither. In a Korean study using the same 2012 KHP data, those having a physician with a place (versus only a place) as a USC had fewer hospital admissions and emergency room visits. ${ }^{6}$ The findings of the above studies are similar to our present ones, except that the other studies used objective indicators of health care service quality, such as readmissions rates, mortality, preventive services use, and hospitalization, whereas our study relied on users' own evaluations.

A study in the United States revealed that having a usual doctor has a greater impact than having a usual site on discretional preventive services, such as blood pressure and cholesterol checkups. ${ }^{20)}$ However, the same study found no statistically significant difference between the two groups in terms of receiving flu shots, pap smears, and mammograms. In other words, different results were found according to the items of preventive services even in the same populations. Considering that there were additional differences in medical systems and cultures between the United States and Korea, further studies in Korea using indicators that are more objective are required.

In some areas of medical services, having only a usual place might not be as effective as having a usual doctor. The effect of a USC (a doctor or a place) is said to be caused by a sense of trust in a physician and the accumulated knowledge of the doctors at the site that is built through long-term doctor-patient interactions, and/or arise from medical record information being shared by doctors who work at the same medical institution. ${ }^{2,12,27,28)}$ Usual doctors can establish a good doctor-patient relationship and understand patients holistically and humanely. A holistic and humane approach makes it easy for a doctor to build a sense of trust, make correct diagnoses with relatively few laboratory tests, and avoid harmful drug interactions. Additionally, a usual doctor may be more likely to consider not only patients' diseases but also their illnesses by approaching patients with a patient-centered method, which includes dealing with patients' feelings about their sickness, ideas about symptoms, and expectations of doctors, as well as the effect of their symptoms on lives. ${ }^{29)}$ Patient-centered medicine, which is closely related to longitudinality, can affect the perception of health care quality. Shared information obtained through medical records can be as effective as knowledge accumulation in certain aspects of technical care, such as the performance of preventive procedures. However, it cannot be equal to long-term doctor-patient relationship in terms of the recognition of patients' problems, judgment of the need for diagnostic interventions, and assessment of the relative merits of different modes of intervention. ${ }^{2)}$

This study has several limitations. First, the presence or absence of a usual place or a usual doctor over the course of a year was determined by individuals' self-reported answers, instead of objective statistics, such as continuity indices. Although the definition of a USC includes 
subjective components, further studies can reveal possible mechanisms of the association between having a usual doctor and patients' higher perception for overall health care services quality. Second, the logistic regression model's discriminative ability (C statistics) to analyze the association between having a usual doctor and individuals' ratings of overall health care quality was low $(<0.7)$. This might be due to limitations of the model, such as remaining confounders (e.g., insufficient number of predictors or categorized/collapsed variables for simplicity). Third, we could not identify cause-and-effect associations as our study had a cross-sectional design. Indeed, there might be reverse causation. Those who assess the service of a doctor or a medical institution highly might choose it as their usual doctor or place. Further studies using a longitudinal design will be possible as the KHP data accumulate over the years.

In conclusion, people with a usual doctor rated overall health care service quality higher compared with those without a USC or with only a place as a USC. This finding implies that new health policies are needed to encourage people in Korea to have a usual doctor.

\section{CONFLICT OF INTEREST}

No potential conflict of interest relevant to this article was reported.

\section{AUTHOR CONTRIBUTION}

Conception and design: Sung NJ, Lee JH; data analysis: Sung NJ; interpretation of data: Sung NJ, Lee JH; writing of the manuscript: Sung NJ; and final approval of manuscript: Sung NJ, Lee JH.

\section{ORCID}

Nak-Jin Sung: https://orcid.org/0000-0002-7965-3965

Jae-Ho Lee: https://orcid.org/0000-0001-5184-7437

\section{REFERENCES}

1. Starfield B. Is primary care essential? Lancet 1994;344:1129-33.

2. Starfield B. Primary care: balancing health needs, services, and technology. New York (NY): Oxford University Press; 1998.

3. Starfield B, Shi L. The medical home, access to care, and insurance: a review of evidence. Pediatrics 2004;113(5 Suppl):1493-8.

4. Blewett LA, Johnson PJ, Lee B, Scal PB. When a usual source of care and usual provider matter: adult prevention and screening services. J Gen Intern Med 2008;23:1354-60.

5. DeVoe JE, Tillotson CJ, Wallace LS, Lesko SE, Pandhi N. Is health insurance enough?: a usual source of care may be more important to ensure a child receives preventive health counseling. Matern Child Health J 2012;16:306-15.

6. Kim KM, Jeon H, Lee JH. Having a physician rather than a place as a usual source of care would be better: from 2012 Korea Health Panel data. J Korean Med Sci 2017;32:4-12.

7. Jung M, Lee KH, Choi M. Perceived service quality among outpatients visiting hospitals and clinics and their willingness to re-utilize the same medical institutions. J Prev Med Public Health 2009;42:151-9.

8. World Health Organization. Quality of care: a process for making strategic choices in health systems [Internet]. Geneva: World Health Organization; 2006 [cited 2017 Jul 9]. Available from: http://www.who.int/ management/quality/assurance/QualityCare_B. Def.pdf.

9. Campbell SM, Roland MO, Buetow SA. Defining quality of care. Soc Sci Med 2000;51:1611-25.

10. Tsai J, Shi L, Yu WL, Lebrun LA. Usual source of care and the quality of medical care experiences: a cross-sectional survey of patients from a Taiwanese community. Med Care 2010;48:628-34.

11. Du Z, Liao Y, Chen CC, Hao Y, Hu R. Usual source of care and the quality of primary care: a survey of patients in Guangdong province, China. Int J Equity Health 2015;14:60.

12. Finney Rutten LJ, Agunwamba AA, Beckjord E, Hesse BW, Moser RP, Arora NK. The relation between having a usual source of care and ratings of care quality: does patient-centered communication play a role? J Health Commun 2015;20:759-65.

13. Kim JH, Cho HJ. Effects of having regular source of care on preventive services and disease control. J Korean Acad Fam Med 2007;28:278-85.

14. An AR, Kim K, Lee JH, Sung NJ, Lee SI, Hyun MK. Having a usual source of care and its associated factors in Korean adults: a cross-sectional study of the 2012 Korea Health Panel Survey. BMC Fam Pract 2016;17:167.

15. Cheng TM. Taiwan's health care system: the next 20 years [Internet]. Washington (DC): Bookings Institution; 2015 [cited 2017 Jul 9]. Available from: https://www.brookings.edu/opinions/taiwans-healthcare-system-the-next-20-years/.

16. Polsa P, Fuxiang W, Saaksjarvi M, Shuyuan P. Cultural values and health service quality in China. Int J Health Care Qual Assur 2013;26: 55-73.

17. Goldstein RB, Rotheram-Borus MJ, Johnson MO, Weinhardt LS, Remien RH, Lightfoot M, et al. Insurance coverage, usual source of care, and receipt of clinically indicated care for comorbid conditions among adults living with human immunodeficiency virus. Med Care 2005;43: 401-10.

18. National Health Insurance Service, Korea Institute for Health and Social Affairs. Report 2012 (I) on the basic analysis of the Korea Health Panel [Internet]. Sejong: Korea Institute for Health and Social Affairs; 2014 [cited 2017 Jul 9]. Available from: https://www.khp.re.kr:444/.

19. Mainous AG 3rd, Gill JM. The importance of continuity of care in the likelihood of future hospitalization: is site of care equivalent to a primary clinician? Am J Public Health 1998;88:1539-41.

20. Xu KT. Usual source of care in preventive service use: a regular doctor versus a regular site. Health Serv Res 2002;37:1509-29.

21. Lebrun-Harris LA, Shi L, Zhu J, Burke MT, Sripipatana A, Ngo-Metzger Q. Effects of patient-centered medical home attributes on patients' perceptions of quality in federally supported health centers. Ann Fam Med 2013;11:508-16.

22. Atlas SJ, Grant RW, Ferris TG, Chang Y, Barry MJ. Patient-physician connectedness and quality of primary care. Ann Intern Med 2009;150: 325-35.

23. Korea Institute for Health and Social Affairs, National Health Insurance Services. 2008-2012 Korea Health Panel annual data codebook [Internet]. Sejong: Korea Institute for Health and Social Affairs; 2012 
[cited 2017 Jul 9]. Available from: https://www.khp.re.kr:444/.

24. Kim YS, Byon LN. Calculation method of household's out-of-pocket expenses for health based on equivalence scales. Korean J Health Econ Policy 2009;15:64-93.

25. Pencina MJ, D'Agostino RB Sr. Evaluating discrimination of risk prediction models: the C statistic. JAMA 2015;314:1063-4.

26. Schoen C, Osborn R, Doty MM, Bishop M, Peugh J, Murukutla N. Toward higher-performance health systems: adults' health care experiences in seven countries, 2007. Health Aff (Millwood) 2007;26:w71734.
27. Weiss LJ, Blustein J. Faithful patients: the effect of long-term physicianpatient relationships on the costs and use of health care by older Americans. Am J Public Health 1996;86:1742-7.

28. Rappaport SH. Supporting the "clinic without walls" with an event-directed messaging system integrated into an electronic medical record. Proc AMIA Annu Fall Symp 1996:648-52.

29. Stewart M, Brown JB, Weston WW, McWhinney IR, McWilliam CL, Freeman TR. Patient-centered medicine: transforming the clinical method. 2nd ed. Abingdon: Radcliffe Medical Press; 2003. 